\title{
Microstructure, mechanical properties and in vitro bioactivity of akermanite scaffolds fabricated by laser sintering
}

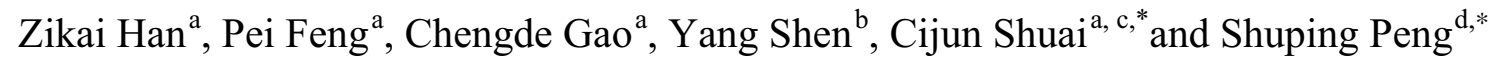 \\ ${ }^{a}$ State Key Laboratory of High Performance Complex Manufacturing, Central South University, \\ Changsha, 410083, P.R. China \\ ${ }^{b}$ State Key Laboratory of New Ceramic and Fine Processing, Tsinghua University, Beijing, 100084 , \\ P.R. China \\ ${ }^{c}$ Shenzhen Research Institute, Central South University, Shenzhen, 518057, P.R. China \\ ${ }^{d}$ Cancer Research Institute, Central South University, Changsha, 410078, P.R. China
}

\begin{abstract}
Akermanite had attracted great attention due to the favourable mechanical properties and excellent biological performance. In this research, the microstructure and mechanical properties of akermanite scaffolds fabricated via laser sintering under different process conditions were studied and characterized. The results showed that the akermanite particles gradually mixed together, grew up and reached complete densification with the scanning speed decreasing from 450 to $150 \mathrm{~mm} / \mathrm{min}$, while micro defects such as air holes occurred at $50 \mathrm{~mm} / \mathrm{min}$. simultaneously, the compressive strength of the scaffolds went up and then descended, and the optimum value was $5.92 \pm 0.41 \mathrm{MPa}$. The Vickers hardness and fracture toughness increased consistently and then tended to stabilize. X-ray diffraction (XRD) results indicated no new phase appeared under all process conditions. MG-63 cell culture revealed that cell adhesion and proliferation occurred, indicating excellent cytocompatibility of the scaffolds. Moreover, in vitro bioactivity tests showed that the apatite layer formed on the scaffolds and became dense and thick with the increase of soaking time in simulated body fluid (SBF), and this fact was further confirmed by energydispersive spectroscopy (EDS).
\end{abstract}

Keywords: Akermanite, laser sintering, mechanical properties, scaffold

\section{Introduction}

Bone tissue engineering was proposed as a promising approach for the repair and regeneration of bone defects. Three-dimensional porous scaffolds played a crucial role in the bone tissue engineering. They could serve as a temporary support for cell growth and facilitate the transportation of nutrients and waste products [1]. In general, the ideal scaffolds should possess interconnected porous structures, excellent biological functions and proper mechanical properties [2]. Traditional scaffold fabrication

\footnotetext{
${ }^{*}$ Corresponding authors: Cijun Shuai, State Key Laboratory of High Performance Complex Manufacturing, Central South University, Changsha, 410083, P.R. China. Tel.: +86 731 88879351; Fax:+86 731 88879044; Email: shuai@csu.edu.cn.

Shuping Peng, Cancer Research Institute, Central South University, Changsha, 410078, P.R. China. Tel.: +86 731 84805412; Fax: +86 731 88879044; Email: shuping@csu.edu.cn.
} 
methods, including gas foaming, fiber bonding and phase separation, are incompetent to accurately control the pore morphology, pore size and overall porosity [3]. To better control the pore structure, selective laser sintering (SLS) technique, as a CAD-based rapid prototyping method, had been developed in recent past, which has advantage of excellent spatial control over architecture.

Akermanite $\left(\mathrm{Ca}_{2} \mathrm{MgSi}_{2} \mathrm{O}_{7}\right)$, a representative of newly accepted calcium silicate based bioceramic, had received considerable attention, as it possesses not only favourable mechanical properties but also superior bioactivity which had been confirmed by the adhesion of osteoblast cells and periodontal ligament cells (PDLCs) in prior studies [4]. Besides, the active effect of ion release, it was also highlighted due to the existence of $\mathrm{Si}$ and $\mathrm{Mg}$ elements in akermanite [5]. Moreover, moderate degradation of akermanite was conducive to cell attachments in vitro and in vivo [6]. Some researchers had reported the fabrication of akermanite scaffolds. Wu et al. prepared akermanite compact with compressionsintering method and found that the fracture toughness of the samples reached $1.83 \mathrm{MPa}^{\circ} \mathrm{m}^{1 / 2}$, which exceeded the required range of cancellous bone and approached the demanding range of cortical bone [7]. Akermanite scaffold with high porosity was prepared by polymer sponge method as well [8].

In this study, the microstructure of akermanite scaffolds sintered under various process conditions was tested with scanning electron microscopy (SEM) and XRD. The effects of scanning speed on mechanical properties were evaluated in terms of compressive strength, Vickers hardness and fracture toughness. The cytocompatibility was assessed by seeding MG-63 cells on the scaffolds. In addition, the apatite layer deposition ability was identified by immersing the scaffolds in SBF

\section{Material and methods}

\subsection{Material and processing}

The akermanite powder (size: $5-40 \mu \mathrm{m}$, purity: $>98 \%$ ) was purchased from the Kun Shan Chinese Technology New Materials Co. Ltd. Briefly, the akermanite powder was prepared by the sol-gel process using TEOS, $\mathrm{Mg}\left(\mathrm{NO}_{3}\right)_{2} \cdot 6 \mathrm{H}_{2} \mathrm{O}$ and $\mathrm{Ca}\left(\mathrm{NO}_{3}\right)_{2} \bullet 4 \mathrm{H}_{2} \mathrm{O}$ as raw materials, then the drying and calcining processes were carried out [9]. The akermanite scaffolds were prepared by using a home-made selective laser sintering system, which had been reported in our previous research [10]. The variation of scanning speed $(450,350,250,150,50 \mathrm{~mm} / \mathrm{min})$ was studied in this paper and other parameters were kept unchanged as followed: laser power: $8.0 \mathrm{~W}$, laser spot diameter: $1.0 \mathrm{~mm}$, scan spacing: 1.5 $\mathrm{mm}$, layer thickness: $0.15 \mathrm{~mm}$, part bed temperature: $80^{\circ} \mathrm{C}$. Besides, in order to estimate the correlation between local sintering temperature and applied specific laser scanning speeds, a three dimensional thermal model for SLS of akermanite powder had been developed based on finite element software (Ansys). The detailed model building, meshing and simulating was described in our previous paper [11]. The highest temperatures located at the spot center were 943, 1025, 1174, 1252 and $1368^{\circ} \mathrm{C}$ at the scanning speed $450,350,250,150$ and $50 \mathrm{~mm} / \mathrm{min}$, respectively.

\subsection{Characterization}

The bulk density and open porosity of the porous scaffold were measured by water displacement using the Archimedes method. The scaffold was about $15 \mathrm{~mm} \times 15 \mathrm{~mm} \times 7 \mathrm{~mm}$ (width $\times$ length $\times$ height). The struts of the scaffolds had a width of $1.1 \mathrm{~mm}$ and a length of $15 \mathrm{~mm}$. Besides, the pores had a size range of 1.5-1.6 mm. So the total volume was $1575 \mathrm{~mm}^{3}$. The actual volume was measured in water, which was $663 \mathrm{~mm}^{3}$. The open porosity was calculated to be $57.9 \%$ and the bulk density reached a 
highest value of $1.14 \mathrm{~g} / \mathrm{cm}^{3}$ at the scanning speed of $150 \mathrm{~mm} / \mathrm{min}$. Besides, the total porosity was measured by gravimetry according to the equation: $\mathrm{P}_{\mathrm{t}}=1-\rho_{\mathrm{s}} / \rho_{\mathrm{m}}$, where $\rho_{\mathrm{s}}$ means the bulk density of the scaffold, which was measured by dividing the weight by the volume of scaffold, and $\rho_{\mathrm{m}}$ means the theoretical density of the material (synthetic akermanite $=2.944 \mathrm{~g} / \mathrm{cm}^{3}$ ). The resulting total porosity was $61.2 \%$ at $150 \mathrm{~mm} / \mathrm{min}$, which was the lowest among all values.

The compressive strength of scaffolds $(15 \mathrm{~mm} \times 15 \mathrm{~mm} \times 7 \mathrm{~mm})$ was measured by using a universal mechanical tester (Shanghai Zhuoji Instruments Co., Ltd, China). The compression test was carried out at a crosshead speed of $0.5 \mathrm{~mm} / \mathrm{min}$ and the stress versus strain curve of the scaffolds was recorded during the tests. The compressive strength was then determined from the load at the point of sample failure through the stress-strain curves. Five samples per processing condition were tested, and the mean and standard deviations were calculated. Besides, the fracture toughness and hardness of the strut specimens were tested by the Vickers microindenter (HXD-1000TM/LCD, Digital Micro Hardness Tester, Shanghai Taiming Optical Instrument Co., Ltd, China). The specimens ( $3 \mathrm{~mm} \times 1.6$ $\mathrm{mm} \times 0.8 \mathrm{~mm}$ ) were embedded in bakelite vertically by a metallographic specimen inlaying machine (XQ-2B, Ф22*15). Then, the surface of the specimens was mechanically polished with 2000-grit emery papers to achieve certain flatness and subjected to indentation. A load of $2.942 \mathrm{~N}$ (300 gf) was exerted on the samples. The fracture toughness $\mathrm{K}_{\mathrm{ic}}$ was calculated by the equation: $\mathrm{K}_{\mathrm{ic}}=0.0824 P \mathrm{c}^{-3 / 2}$, where $P$ is the indentation load and $c$ is the length of the induced crack. Similarly, five specimens per each condition were tested.

The microstructure was investigated by SEM and EDS. Briefly, the samples were trimmed with a razor blade and the surface was coated with gold by a sputter coater (JEOL, JFC-1300). During the gold coating process, the gas pressure was stabilized at $50 \mathrm{~m}$ Torr and the current was set at $20 \mathrm{~mA}$ for 120 s. SEM image analysis and element analysis were performed by A JEOL JSM-6490LV microscope equipped with an EDS detector at an acceleration voltage of $20.0 \mathrm{kV}$. Besides, the phase compositions of raw and sintered akermanite were analyzed by XRD (D8-ADVANCE, Bruker Co. German) at a scanning rate of $0.02^{\circ} / \mathrm{s}$ in $2 \theta$ range from $25^{\circ}$ to $40^{\circ}$ using $\mathrm{CuKa}$ radiation.

\subsection{Cell culture and bioactivity test}

MG-63 osteoblast-like cells were used to investigate cytocompatibility of the scaffolds. The cells were purchased from American Type Culture (ATCC, Rockville, MD) and maintained in DMEM/10\% FBS and $50 \mu \mathrm{g} / \mathrm{ml}$ streptomycin in $5 \% \mathrm{CO}_{2}$ at $37^{\circ} \mathrm{C}$. The strut samples $(10 \mathrm{~mm} \times 1.1 \mathrm{~mm} \times 0.6 \mathrm{~mm})$ were prepared and soaked in $70 \%$ ethanol for $3 \mathrm{~h}$. After soaking, the rinse process was repeated thrice in PBS buffer solution. Then MG-63 cells seeded in 12-well plates with the samples at a density of $1 \times 10^{5}$ cells/well and the plates were incubated at $37^{\circ} \mathrm{C}$ in $5 \% \mathrm{CO}_{2}$. Subsequently, the cell-seeded samples were kept at $37^{\circ} \mathrm{C}$ in a humidified incubator under standard conditions. The culture time was set to 1,5 and 9 days and the medium was replaced every second day. After given time, the samples were washed with PBS and dehydrated with gradient series of ethanol concentration: 30\%, 50\%, 70\%, 90\% and $100 \%(\mathrm{v} / \mathrm{v})$. Besides, in vitro bioactivity of scaffolds was carried out in SBF solution. The SBF medium was prepared according to Kokubo's protocol [12]. The scaffolds $(15 \mathrm{~mm} \times 15 \mathrm{~mm} \times 7 \mathrm{~mm})$ were then immersed in $100 \mathrm{ml}$ of SBF solution at $37^{\circ} \mathrm{C}$ in a shaking water bath $(100 \mathrm{rpm})$ for $7,14,21$ and 28 days. After the stipulated time, the scaffolds were collected from SBF medium, rinsed with distilled water and dried in air at room temperature. The evolution of the surface and cross-section was visualized by SEM. The formation of apatite layer was confirmed by EDS. Besides, the rate of weight loss $\left(\mathrm{W}_{\mathrm{L}}\right)$ was also calculated according to the formula: $\mathrm{W}_{\mathrm{L}}=\left(\mathrm{W}_{0}-\mathrm{W}_{1}\right) / \mathrm{W}_{0} \times 100 \%$, where $\mathrm{W}_{0}$ and $\mathrm{W}_{1}$ denote the weight of the scaffolds before and after soaking, respectively. 


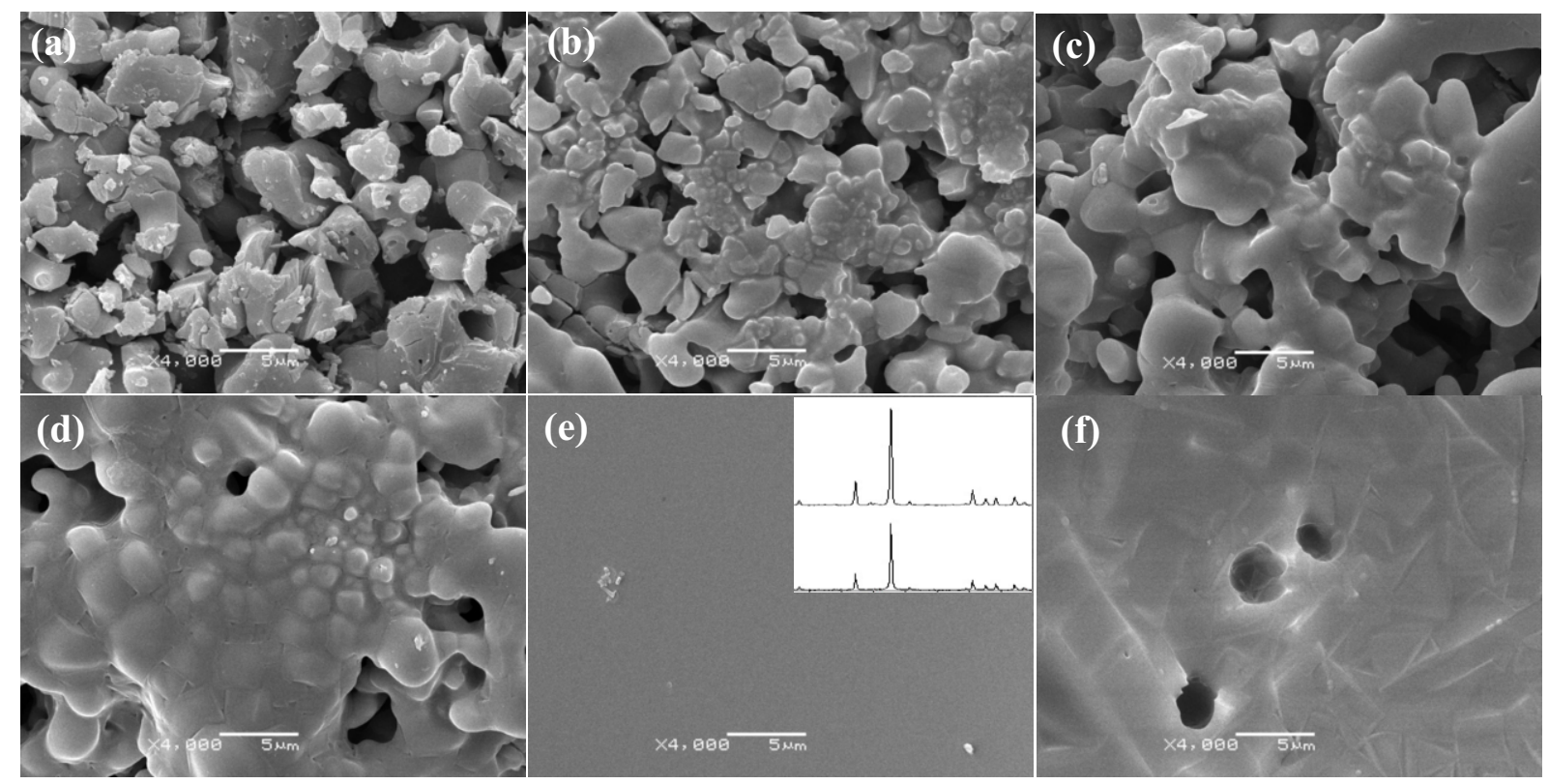

Fig. 1. SEM micrographs of the akermanite scaffolds sintered at various scanning speed: (a) unsintered powder; (b) 450 $\mathrm{mm} / \mathrm{min}$, (c) $350 \mathrm{~mm} / \mathrm{min}$, (d) $250 \mathrm{~mm} / \mathrm{min}$, (e) $150 \mathrm{~mm} / \mathrm{min}$, (f) $50 \mathrm{~mm} / \mathrm{min}$.

\section{Results and discussions}

\subsection{Microstructure evolution}

The microstructure of unsintered and sintered akermanite at different scanning speed was shown in Figure 1 . The unsintered akermanite particles were irregularly shaped and distributed randomly (Figure 1(a)). At the scanning speed of $450 \mathrm{~mm} / \mathrm{min}$, the particles began to close together and necks between the agglomerates began to form partially (Figure 1(b)). Interagglomerate shapeless pores could be observed easily and necks between agglomerates became even stronger when the scanning speed decreased to $350 \mathrm{~mm} / \mathrm{min}$ (Figure 1(c)). A great number of pores disappeared at the speed of 250 $\mathrm{mm} / \mathrm{min}$ (Figure 1(d)). The surface of scaffolds was smooth and flattened when the speed was $150 \mathrm{~mm} / \mathrm{min}$ (Figure 1(e)), while obvious air holes emerged when the speed decreased to $50 \mathrm{~mm} / \mathrm{min}$ (Figure 1(f)). The reason for the appearance of air holes was that: the sintering temperature increased due to the decrease of the scanning speed, and the driving force for sintering reaction was improved, which led to the rapid grain boundary migration rate. The rate was faster than the release of air bubbles, and thus some bubbles maintained in the substrate [13]. Besides, the phase compositions of unsintered and sintered akermanite were characterized by XRD (Figure 1(e)). No additional peaks formed for sintered samples, indicating that no new phases and phase transformation had occurred, revealing that the XRD patterns were consistent with the standard PDF card (JCPD file No.83-1815).

\subsection{Mechanical properties}

The compressive strength, Vickers hardness and fracture toughness of the scaffolds were tested. It was shown that the compressive strength was $0.96 \pm 0.12 \mathrm{MPa}$ for scaffolds sintered at the speed of 450 
$\mathrm{mm} / \mathrm{min}$, and then increased significantly when the scanning speed decreased (Figure 2(a)). The optimum compressive strength was $5.92 \pm 0.41 \mathrm{MPa}$ at the speed of $150 \mathrm{~mm} / \mathrm{min}$. However, the compressive strength decreased to $4.97 \pm 0.32 \mathrm{MPa}$ with a further decrease in scanning speed to $50 \mathrm{~mm} / \mathrm{min}$. Meanwhile, the Vickers hardness was $2.83 \pm 0.25 \mathrm{GPa}$ at $450 \mathrm{~mm} / \mathrm{min}$, and then increased to $4.60 \pm 0.29$ $\mathrm{GPa}$ at $350 \mathrm{~mm} / \mathrm{min}$. After that, Vickers hardness increased slightly from 350 to $50 \mathrm{~mm} / \mathrm{min}$ and the peak value was $6.19 \pm 0.36 \mathrm{GPa}$ at $50 \mathrm{~mm} / \mathrm{min}$ (Figure 2(b)). The fracture toughness increased from $0.63 \pm 0.11$ to $1.72 \pm 0.20 \mathrm{MPa} \bullet \mathrm{m}^{1 / 2}$ when the scanning speed decreased from 450 to $50 \mathrm{~mm} / \mathrm{min}$ (Figure 2(c)). In general, many researches have carried out research on the mechanical properties of akermanite scaffolds. The compressive strength of the akermanite scaffolds fabricated with polymer sponge method by $\mathrm{Wu}$ was relatively low [8]. The optimum compressive strength of the scaffold with a porosity of $63.5 \%$ was lower than that of ours with a similar porosity of $57.9 \%$. The research cited above showed compromised mechanical properties compared with ours. This fact could be attributed to the selection and analysis of the optimum process parameter.

\subsection{Cell culture and bioactivity tests}

The scaffolds with favorable mechanical properties, which were fabricated at the scanning speed of $150 \mathrm{~mm} / \mathrm{min}$, were used in cell culture and in vitro bioactivity tests. The adhesion and proliferation of MG-63 cells on the scaffolds were observed by SEM after different time. Lamellipodia outgrowth was clearly visible and some cytoplasm spread at the boundary of processes for 1 day, indicating the formation of focal adhesion which favored further cell spreading (Figure 3(a)). Cells appeared to stack in layers and stretched out to form aggregates between each other for 5 days. Not only lamellipodia but also filopodia were observed apparently (Figure 3(b)). After 9 days, most cells had grown into a continuous thick cellular layer covered the surface completely (Figure 3(c)).
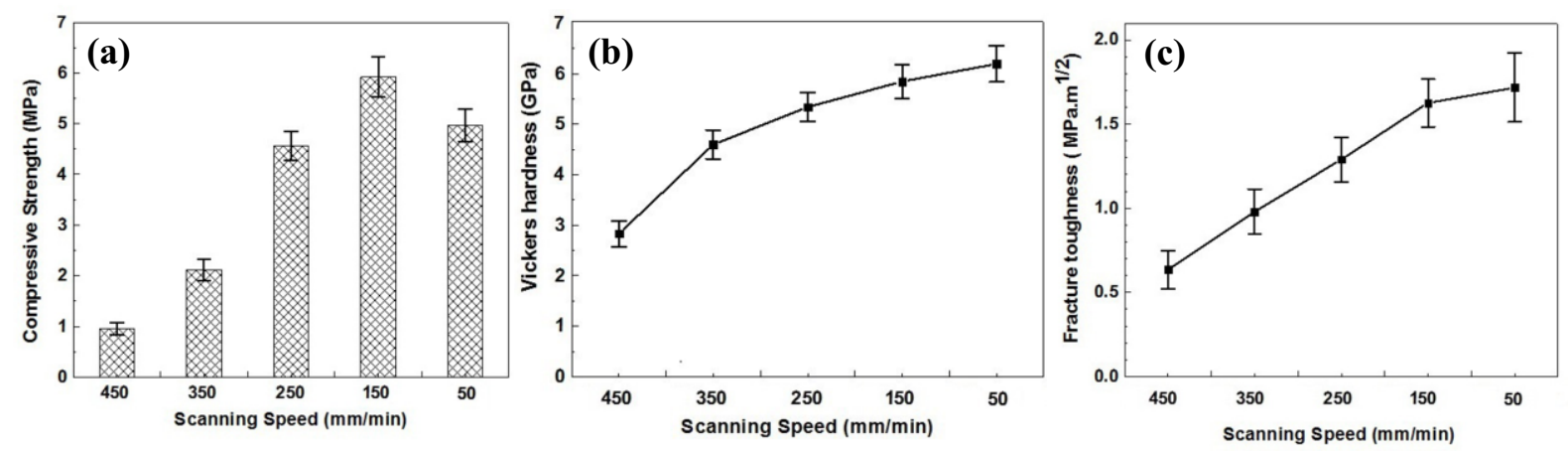

Fig. 2. Effect of scanning speed on (a) compressive strength, (b) Vickers hardness and (c) fracture toughness of porous akermanite scaffolds $(\mathrm{n}=5, \mathrm{p}<0.05)$. 

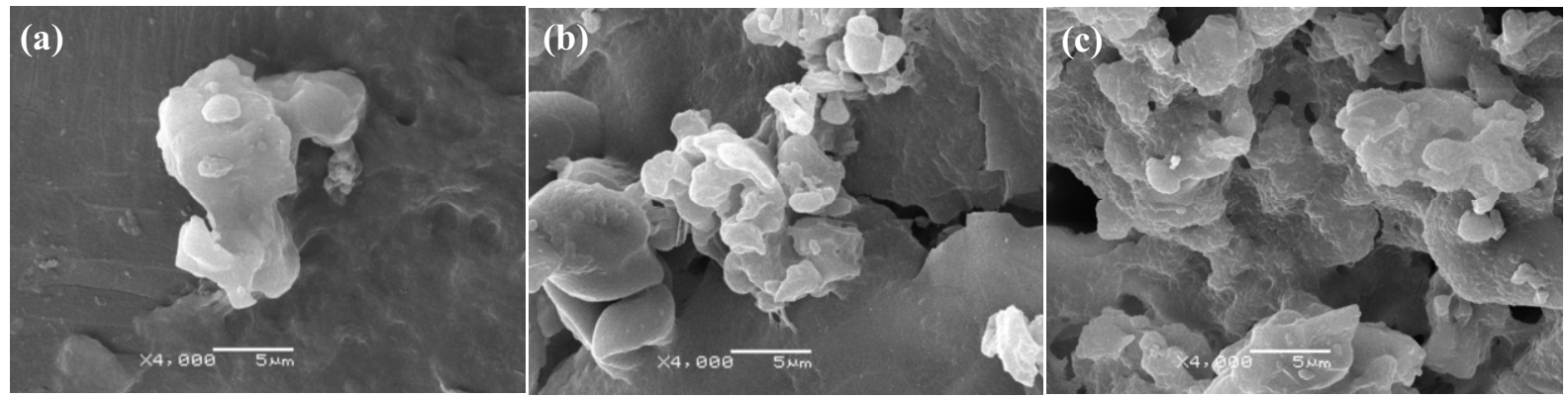

Fig. 3. MG-63 morphology on the surface of akermanite scaffolds after (a) 1 day, (b) 5 days and (c) 9 days.
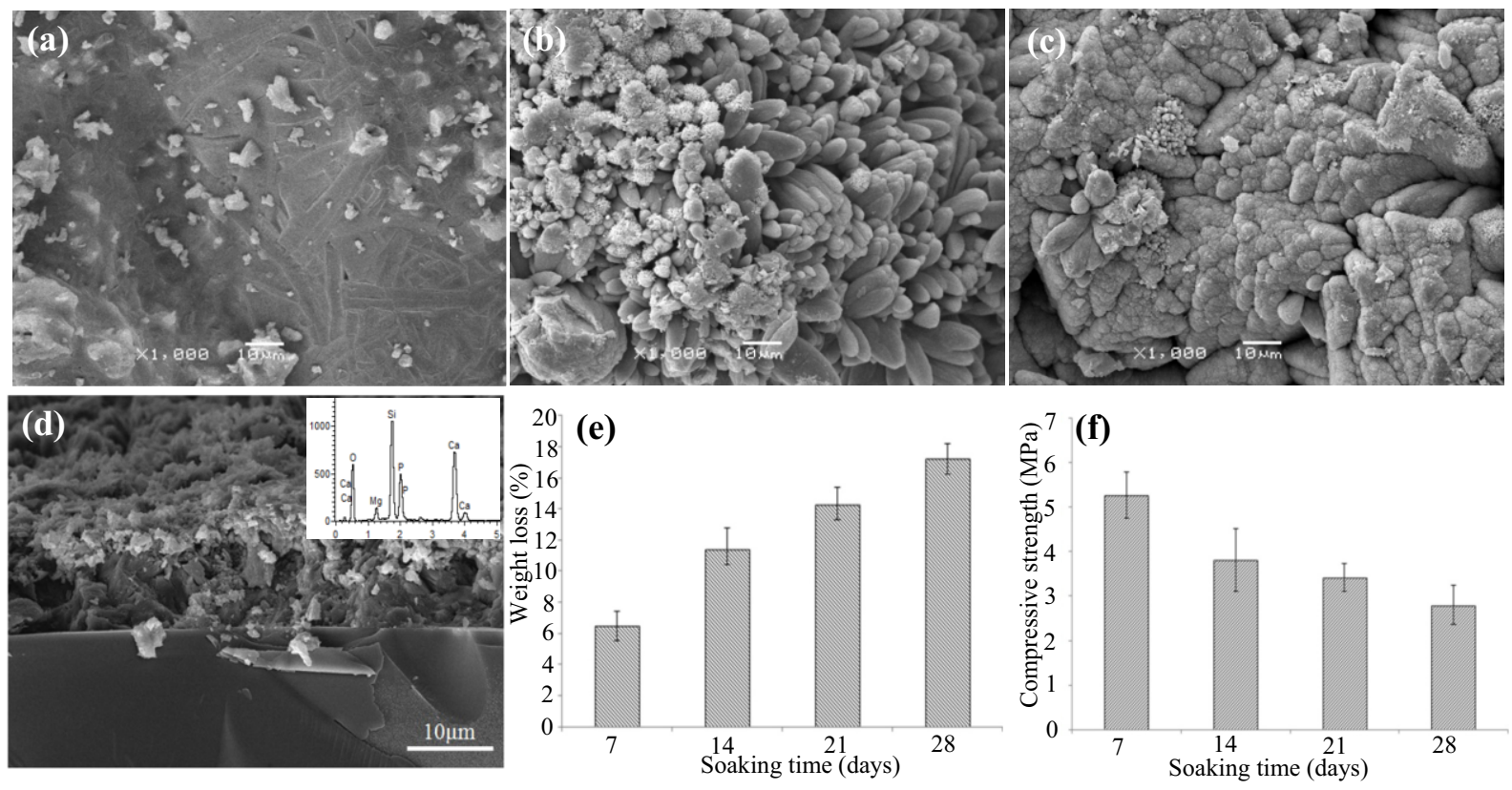

Fig. 4. SEM micrographs of the scaffolds after immersing in SBF for (a) 7 days, (b) 14 days and (c) 21 days. (d) SEM micrographs of the cross-section of the scaffolds after soaking in SBF for 21 days. (e) Weight loss and (f) compressive strength of the scaffolds after soaking in SBF for various time.

The bioactivity tests were performed in SBF for 7, 14 and 21 days (Figure 4). A few apatite deposition began to form after being soaked in SBF for 7 days (Figure 4(a)). Apatite deposition formed on the surface of shuttle-like precipitation when the scaffolds were soaked for 14 days (Figure 4(b)). The apatite particles became more and more, forming a continuous layer covering the entire substrate for 21 days (Figure 4(c)). The cross-section of the scaffolds after soaking in SBF for 21 days was also observed to evaluate the thickness of formed apatite layers (Figure 4(d)). It was clear that the thickness reached about $13 \mu \mathrm{m}$ after 21 days. EDS analysis further confirmed that the $\mathrm{Ca} / \mathrm{P}$ molar ratio was 1.74 for the deposition coated on the substrate, which was close to 1.67 for HAP. Besides, the results presented in Figure 4(e) showed that the weight loss increased continuously over the entire incubation period and reached $17 \%$ after 28 days. Besides, the compressive strength of the scaffolds after soaking in SBF was depicted in Figure 4(f). The results showed that the compressive decreased as soaking 
time increased due to degradation. Moreover, the decreasing rate was only $34 \%$ after 14 days, which was lower than the strength of hydroxyapatite scaffolds after immersion [14].

\section{Conclusion}

The relationship between process parameters, microstructure and mechanical properties of the scaffolds fabricated under various process conditions was investigated. The results showed that the structure of the sintered akermanite became denser when the scanning speed decreased from 450 to 150 $\mathrm{mm} / \mathrm{min}$, while some micro defects occurred as the scanning speed further decreased. Meanwhile, the compressive strength went up and then descended with the decrease of the scanning speed. The optimum compressive strength was $5.92 \pm 0.41 \mathrm{MPa}$ at $150 \mathrm{~mm} / \mathrm{min}$. And MG-63 cells attached and spread well on the scaffolds, and proliferated with the increasing culture time. Moreover, the scaffolds also exhibited reliable mechanical stability after soaking in SBF. Apatite deposition formed on the surface of the scaffolds, which indicated good bioactivity. The akermanite scaffolds fabricated via SLS might be an excellent candidate for bone tissue applications.

\section{Acknowledgement}

This work was supported by the following funds: (1) The Natural Science Foundation of China (51222506, 81000972); (2) Hunan Provincial Natural Science Foundation of China (14JJ1006); (3) Project supported by the Fok Ying-Tong Education Foundation, China (131050); (4) Shenzhen Strategic Emerging Industrial Development Funds (JCYJ20130401160614372); (5) The Open-End Fund for the Valuable and Precision Instruments of Central South University; (6)The faculty research grant of Central South University (2013JSJJ011, 2013JSJJ046) and (7) State Key Laboratory of New Ceramic and Fine Processing, Tsinghua University (KF201413)

\section{References}

[1] L. Soletti, Y. Hong, J. Guan and J.J. Stankus, A bilayered elastomeric scaffold for tissue engineering of small diameter vascular grafts, Acta Biomaterialia 6 (2010), 110-122.

[2] D. Depana, B. Girasea, J.S. Shahb and R.D.K. Misraa, Structure-process-property relationship of the polar graphene oxide-mediated cellular response and stimulated growth of osteoblasts on hybrid chitosan network structure nanocomposite scaffolds, Acta Biomaterialia 7 (2011), 3432-3445.

[3] L. Zhao, V.K. Lee, S.S. Yoo, G. Dai and X. Intes, The integration of 3-D cell printing and mesoscopic fluorescence molecular tomography of vascular constructs within thick hydrogel scaffolds, Biomaterials 33 (2012), 5325-5332.

[4] E. Bernardo, J.F. Carlotti, P.M. Dias and L. Fiocco, Novel akermanite-based bioceramics from preceramic polymers and oxide fillers, Ceramics International 40 (2014), 1029-1035.

[5] C. Wu and J. Chang, Degradation, bioactivity and cytocompatibility of diopside, akermanite, and bredigite ceramics, Journal of Biomedical Materials Research Part B: Applied Biomaterials 83 (2007), 153-160

[6] M. Razavi, M. Fathi, O. Savabi and S.M. Razavi, Controlling the degradation rate of bioactive magnesium implants by electrophoretic deposition of akermanite coating, Ceramics International 40 (2014), 3865-3872.

[7] C. Wu and J. Chang, A novel akermanite bioceramic: Preparation and characteristics, Journal of Biomaterials Applications 21 (2006), 119-129.

[8] C. Wu, J Chang and W. Zhai, Porous akermanite scaffolds for bone tissue engineering: Preparation, characterization, and in vitro studies, Biomedical Materials Research Part B: Applied Biomaterials 78 (2006), 47-55.

[9] O.M. Goudouri, E. Theodosoglou, E. Kontonasaki and J. Will, Development of highly porous scaffolds based on bioactive silicates for dental tissue engineering 49 (2014), 399-404. 
[10] C. Shuai, Y. Nie and C. Gao, The microstructure evolution of nanohydroxapatite powder sintered for bone tissue engineering, Journal of Experimental Nanoscience 8 (2013), 762-773.

[11] C. Shuai, P Feng, C. Gao and Y. Zhou, Simulation of temperature field during the laser sintering process of nanohydroxyapatite powder, Advanced Materials Research 314 (2011), 626-629.

[12] T. Kokubo and H. Takadama, How useful is SBF in predicting in vivo bone bioactivity? Biomaterials 27 (2006), 29072915.

[13] P.V. Landuyt, F. Li, J.P. Keustermans, J.M. Streydio and F. Delannay, The influence of high sintering temperatures on the mechanical properties of hydroxylapatite, Journal of Materials Science: Materials in Medicine 6 (1995), 8-13.

[14] L. Jiang, Y. Li, X. Wang, L. Zhang, J. Wen and M. Gong, Preparation and properties of nano-hydroxyapatite /chitosan/ carboxymethyl cellulose composite scaffold, Carbohydrate Polymers 74 (2008), 680-684. 